\title{
Consideración de la interacción de tipologías de microarquitectura inmótica con las preexistencias ambientales y con el espacio.
}

\author{
Consideration of the interaction of inmotics microarchitecture tipologies with the environment \\ preexisting conditions and with the space
}

\author{
Hernández, Silvia Patricia \\ FAUD. UNC Argentina \\ arqpatriciahernandez@gmail.com
}

\author{
Lanzone, Luciana \\ FAUD. UNC Argentina \\ lulanzone@gmail.com
}

Landerberg, Raquel

FAUD. UNC Argentina

arqcomercial.rl@gmail.com

\author{
Ruiz, José Manuel \\ FAUD. UNC Argentina \\ interestudio@hotmail.com
}

Rezk, alejandra
FAUD. UNC Argentina
alerezk@yahoo.com.ar

Viecens, Martin

FAUD. UNC Argentina

martinviecens@yahoo.com

\begin{abstract}
We work in the technics and design advantages that are happening in the world, and in Argentina, around microarchitecture, applying these in concrete useful design proposals for urban spaces called intersticiales. Starting from pre-existings conditions of the place, it relation between the landscape, the sounds, and the climate to answer to them with the design. The proposal will be articulated and organized according to the plans and rules from the city of Córdoba. These typologies are designs of inmotic microarchitecture, in this case a health station, just in the entry of the Sarmiento park, a big gym without ceiling.
\end{abstract}

Keywords: Inmótics ; Urban micro-architecture; Pre- existing environmental ; Sustainability.

\section{Introducción}

\section{Domótica. Inmótica}

La evolución histórica indica que en un principio los sistemas aplicados en las construcciones eran de control manual. En los Estados Unidos, tras la crisis petrolera de los años 70, con el objetivo principal de generar un ahorro en el consumo, surgen las primeras automatizaciones en edificios, (Romero Morales et al, 2006). Luego, de las primeras automatizaciones con el uso de los termostatos, se comenzaron a usar los sensores para los controles de humedad, caudal de aire, etc., en los sistemas de climatización, y para los controles de intrusos, en los sistemas de alarmas.

Se la llamó domótica, que proviene de domus: casa y tica: de domotique (del francés): robótica (Enciclopedia Larousse, 1988).

El término domótica tiene una génesis análoga a la del término informática, sustituyendo el prefijo que significa información por otro derivado de la palabra latina domus, que significa casa. También reciben un trato análogo en la bibliografía en lengua inglesa, en la que son más comunes otros términos como computing en lugar de informática 0 smart house e intelligent building en lugar de domótica. (Recuero, Caminos, 1999).

La domótica surge entonces con el objetivo principal de otorgar al usuario el máximo confort y seguridad con la mayor economía y eficiencia energética.

Cuando se orienta a edificios terciarios, no viviendas, estamos ante la presencia de la inmótica, que realiza la gestión de la energía incluyendo las automatizaciones de las actividades y el trabajo. (Morales, Serrano, Lozano, 2006).

Esto incluye la ofimática como un subsistema. La ofimática es el conjunto de técnicas, aplicaciones y herramientas informáticas que se utilizan en funciones de oficina para optimizar, automatizar y mejorar los procedimientos o tareas relacionados. (Computer Science and Communications Dictionary, 2001). 
En este trabajo usaremos el término inmótica, que es la nominación específica para la domótica cuando la tipología no es vivienda y dejaremos el uso del término domótica para las generalidades.

\section{Preexistencias ambientales}

La propuesta de este programa de investigación (SECyT 2016/7), se basa en la consideración de que los espacios arquitectónicos o urbanos interactúan con el ambiente, modificando, aprovechando o sufriendo las preexistencias ambientales. Esta interacción puede darse en múltiples escalas. Para Monroy una adecuada calidad ambiental del hábitat construido se alcanza a partir de un conjunto de técnicas, infraestructuras y equipamientos que en conjunto definen el concepto de acondicionamiento ambiental. (Maristany, 2015, director del programa)

Considerar las preexistencias según Bohigas (1986) es dejar de lado la arquitectura única, la exhibición personalista o desprecio insolidario que fragmenta la ciudad. (Rogers, 1961). Por lo cual adherimos a Hernández (2011) que destaca que la actual preocupación por el deterioro ambiental y por un entorno que sea eficiente en el uso de los recursos debe capitalizarse y apuntar a generar herramientas que permitan afrontar los retos que presenta la sostenibilidad desde un enfoque multidisciplinar, entendiendo el hábitat no solo como el tejido residencial, sino involucrando la red de equipamientos y espacio público que lo integra al resto de la ciudad. (Hernández Achury, 2011)

Trabajaremos la situación real de las condicionantes del lugar con sus pre-existencias como el agua, el clima, el paisaje y el ruido. El ruido está considerado como uno de los factores contaminantes más duros e invisible.

\section{Hipótesis}

Es posible trabajar con tipologías de micro-arquitectura urbana desarrollándolos en propuestas concretas de diseño útil para determinados espacios urbanos llamados residuales o intersticiales, y generar espacios o reinterpretarlos para que sean interactivos, inclusivos y sustentables con tecnología domótica teniendo como condicionantes las preexistencias ambientales. La propuesta será articulada y organizada de acuerdo a los planes y normas que regulan la ciudad de Córdoba.

\section{El Sitio}

Este proyecto es parte de las anteriores investigaciones donde estábamos trabajando espacios intersticiales. Ya teníamos planteado el área, zona plaza España.
La plaza España además de ser un nudo neurálgico, convergen 8 avenidas, tiene en sus inmediaciones 5 museos. Se le suma el parque sarmiento, diseñado por el arquitecto Hugo Thays, por lo que podríamos decir que la plaza y especialmente el sitio elegido, es también la puerta de ingreso al mismo parque.

El Parque Sarmiento es un espacio verde parquizado, el más grande de la ciudad de Córdoba. Está ubicado en el centro geográfico de la ciudad a pocas cuadras del microcentro en una de las zonas de mayor densidad demográfica. Lleva el nombre en honor a Domingo Faustino Sarmiento, ex presidente de la República Argentina.

Es parte del barrio Nueva Córdoba, que tiene 37.000 habitantes (censo 2008), es el sector más densamente poblado de la ciudad, con mayoría de residentes estudiantes. Estos estudiantes en busca de una vida sana, y para distracción utilizan el parque para caminatas, trotar o hacer gimnasia. El parque también cuenta con una pileta pública.

La Ciudad Universitaria está a pocas cuadras de la plaza. Es la Universidad Nacional de Córdoba, cuenta con 13 sedes de distintas facultades, 88 carreras de grado más las 118 de postgrado. Mueve una población de 118.000 alumnos. Este será fundamentalmente nuestro target. Aunque también consideraremos alumnos de otras universidades y escuelas, y ciudadanos que cruzan por este sector para ir a sus viviendas o tomar un colectivo. La vida saludable es para todos.

Es común e intenso el uso deportivo de este espacio, a toda hora se ven cientos de personas disfrutando el gran gimnasio a cielo abierto del parque Sarmiento.

Dentro del parque hay circuitos de salud, con numerosas plazas de ejercicios. Estos circuitos de salud son 8. (imagen1).

\section{SIIIO 2}

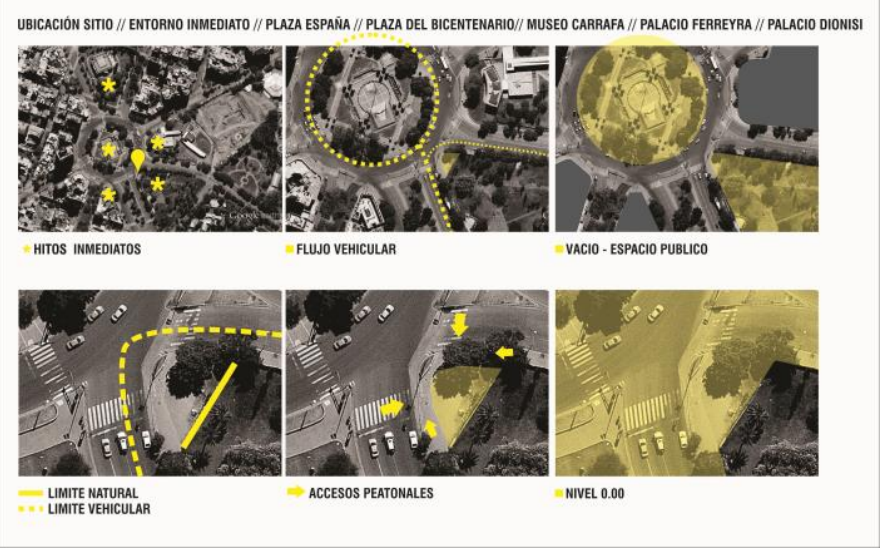

Imagen 1, parque, estaciones, imagen propia 


\section{Espacios residuales, instersticios espaciales}

Reconocemos en la compleja trama urbana de las ciudades espacios llamados residuales que en realidad son instersticios espaciales. Considerando como el espacio que media entre dos partes de distintos objetos o entre dos del mismo cuerpo. La idea de intersticialidad es propia del estudio de las espacialidades urbanas contemporáneas.

Antecedentes en investigaciones de espacios intersticiales permiten definir, asociación de conceptos afines tales como espacio chatarra (Koolhaas; 2002), inbetween (E2 groupe; 2003), terrain vague (De Solá Morales; 1996), áreas de impunidad (Ábalos yHerreros; 1997), no lugar (Augè; 1997), entre otros, que fueron tomados como categorías antecedentes.

En la tarea de aproximarse a nuevas lecturas operativas sobre el territorio, se trabaja a partir de investigar los procesos actuales que intervienen en la espacialidad intersticial en términos de reinterpretación como potencial de nuevas propuestas. Se trata de nuevas configuraciones que se trabajarán con la adaptación, o engrosamiento o completamiento de estos intersticios. Hablamos de intersticio al referirnos a la situación espacial particular y diferenciable determinada por improntas espaciales emergentes de la superposición de nuevas programaciones. La intersticialidad se manifiesta en oposición a la conservación, a la continuidad.

El espacio elegido en contra de lo que dice Deleuze (1980), está ahí esperándonos, como puerta del gimnasio abierto que es el parque. Vacío, dejado, sin tratamiento, alrededor de $100 \mathrm{~m} 2$. El territorio es un acto; eso que se hace al descubrir y al conquistar, el territorio es como resultado de una territorialización. (Deleuze 1980).

Y nosotros al mirarlo, y elegirlo, lo territorializamos.

Es intersticial, porque no se continúa. La plaza de los Aros, llamada Paseo del bicentenario, está arriba, como haciendo barrera; además la vereda peatonal lo circunda, resulta un espacio interplazas.

En estos sitios, parte de las ciudades, interviene el mobiliario urbano. Trabajaremos con equipos urbanos o mobiliarios urbanos, que es todo aquello que amuebla la calle, y con equipamientos urbanos, que son contenedores.
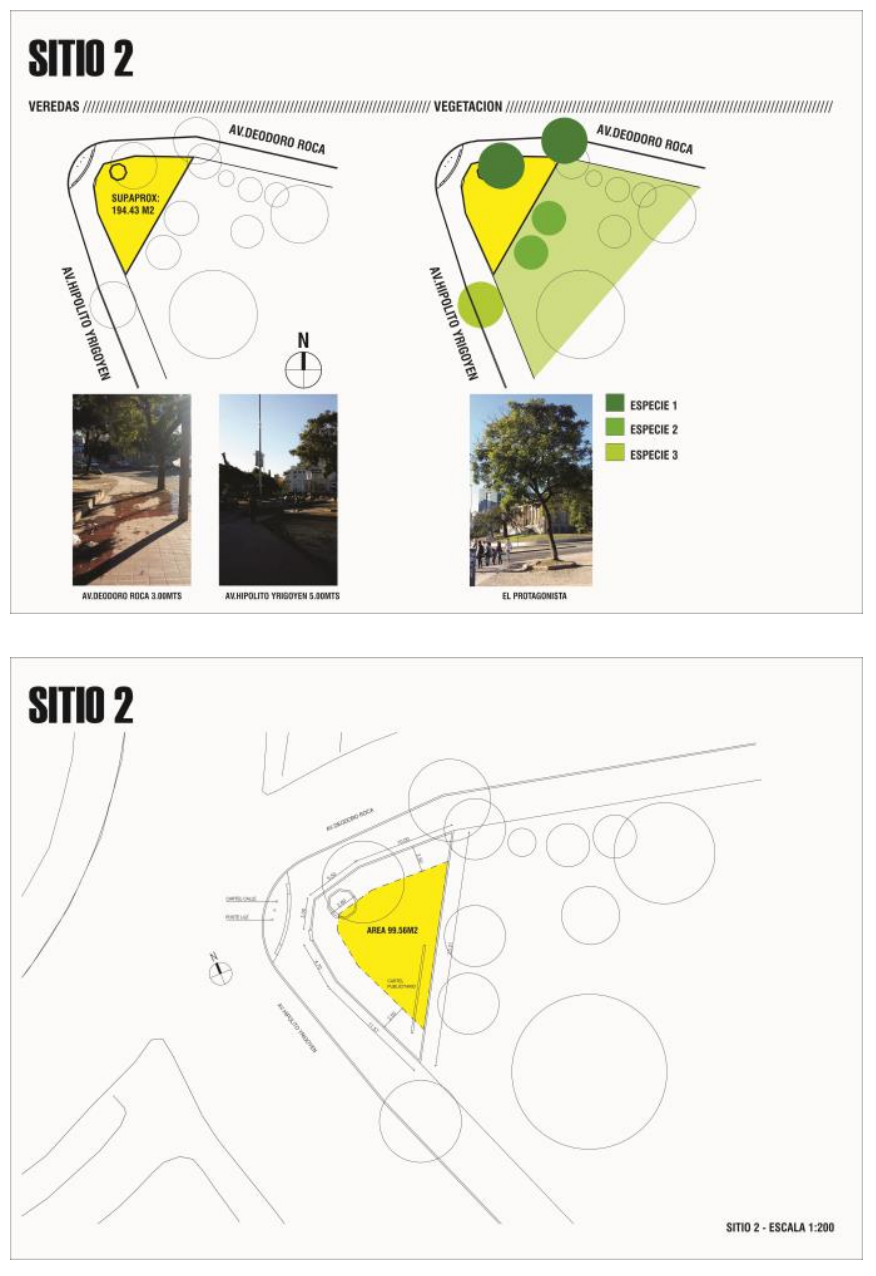

\section{SIIIO 2}

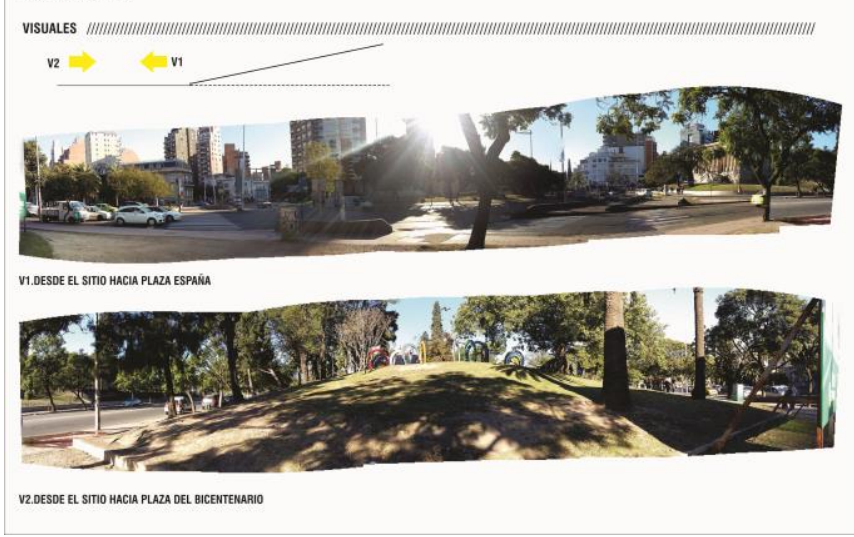

Imagen 2, 3 y 4. Análisis sitio, imágenes propias

Objetivos

1. Elaborar un diagnóstico del estado actual del diseño y producción de micro-arquitectura domótica y sustentable, en tipología desarrolladas en espacios intersticiales, en el mundo y en argentina. 
2. Determinar categorías que permitan configurar los aspectos implicados en cuanto a diseño, prestaciones, confort, economía, seguridad, diseño inclusivo y grado de tecnología aplicada para espacios transformados en útiles.

3. Evaluar los modos de funcionamiento de los equipos domóticos existentes en estas tipologías, en cuanto al modo en que se realiza la automatización, regulación y control de: energía, optimización de la función, redes informáticas, iluminación, climatización, ductilidad, y otras variables y su relación con las centrales de control.

4. Estudiar los comportamientos de los usuarios, la interacción propuesta, las preferencias y rendimientos.

5. Desarrollar tipologías de diseño para la salud, en la puerta del Parque Sarmiento, y de este ejercicio generar pautas que den fundamento a las tendencias de diseño y producción en nuestro país cumpliendo los objetivos de esta investigación, de acuerdo a las demandas del medio y la innovación tecnológica.

6. Realizar un diagnóstico del resultado del comportamiento de los sistemas descriptos en -2 y su impacto en el medio socio- económico y ambiental.

\section{Propuesta}

Se realizó un ensayo de propuesta de diferentes tipologías en relación a la arquitectura y los espacios urbanos, mostrándoles usos y realidades diferentes a los que están acostumbrados, y accediendo a una ciudad mutante con la alternancia del equipamiento que permite diferentes usos para distintos usuarios. Y se eligió una tipología.

\section{Consideraciones generales:}

El mobiliario urbano es todo aquello que amuebla la calle, todo lo que, en el interior de una ciudad o de una aglomeración, se encuentra erigido en el borde de las vías, sobre las aceras, o sobre la misma vía pública.

El equipamiento urbano, es algo más que el mobiliario urbano, ya que contiene, y quizás no está ubicado en la acera, es sostenible cuando se integra con el paisaje y a la vez genera energía limpia a través de paneles solares y/o eólica. Se trata de integrar servicios para la vida urbana y para la naturaleza.

Los elementos urbanos identifican la ciudad y a través de ellos podemos conocer y reconocer las ciudades.

Tenemos como objetivo mejorar el medioambiente, con el tratamiento e intervención de un espacio público, engrandecer con el diseño que conlleva orden y que contempla la función, la morfología y lo sustentable, mediante propuestas concretas de modelos de uso urbanístico y arquitectural y de equipamiento urbano. Se trata de integrar servicios para la vida urbana y para la naturaleza.

El diseño de espacios que ocupen los intersticios urbanos, que responda y se adecue a su entorno, o se destaque, para los usos que la sociedad demanda es una tarea muy comprometida. Para ello es fundamental la comprensión del medio y una lectura clara detenida de su comportamiento.

Con este proyecto hemos querido aceptar un cambio de actitud de la población urbana, ofreciendo posibles soluciones de mobiliario para el espacio urbano que facilitan el uso de las nuevas tecnologías móviles en espacios públicos y el uso de energías alternativas, como la solar, para satisfacer las necesidades de energía.

Coincidimos con Aurelli(2012) cuando dice que los espacios que activan lo común, ya sean estos públicos o privados, de uso particular o colectivo, deberían privar como objetivo la jerarquía de decisiones de lo urbano y lo arquitectónico. ....Cabe aquí insistir en el hecho que mientras un edificio, u otro producto del diseño, es una forma definida y finita, su proceso de producción y su uso, es una realidad que no puede ser reducida a un único objeto o a la autoría de un solo despacho o arquitecto individual.( Aurelli 2012). En esta simple y a la vez contundente afirmación se encierra el origen de la relación social entre la arquitectura y la sociedad en la que se desenvuelve, más aún, como sigue Aurelli, solamente cuando el lenguaje arquitectónico asume en su apariencia estética la realidad de lo común, esta será una verdadera manifestación de una arquitectura potencial.

\section{Hidrostation}

Convencidos de que los beneficios de la gimnasia va más allá del beneficio físico y de salud. La gimnasia nos produce endorfinas y bienestar. La actividad física es una gran promotora de la salud mental y de las habilidades sociales. También favorece la longevidad y protege contra enfermedades.

Desde el punto de vista físico, la O.M.S. (Organización Mundial de la Salud), indica que la realización de actividad física de forma continuada prolonga la longevidad y protege contra el desarrollo de enfermedades. En la actualidad, los nutricionistas recomiendan acompañar las dietas balanceadas con ejercicio regular para complementar el descenso de peso de manera progresiva y saludable. Existe evidencia suficiente que prueba que las personas que llevan una vida físicamente activa, que se ejercitan diariamente, pueden obtener una larga lista de beneficios para su salud:

- Mejora del aspecto estético, permite estar en forma; aumenta la masa muscular; favorece la flexibilidad y coordinación; disminuye la sensación de cansancio y genera sensación de mayor nivel de energía. 
- Mejora la resistencia y condición física, incrementando la capacidad funcional para realizar otras actividades físicas de la vida diaria.

- El fortalecimiento de los huesos disminuye el riesgo de lesiones o trastornos como la osteoporosis.

- $\quad$ Previene o reduce el riesgo de padecer enfermedades, como las cardiovasculares, ya que disminuye el colesterol malo y aumenta el bueno, protege las arterias, previene el riesgo de infarto y disminuye la presión alta; O la obesidad, presión alta, diabetes, artritis y algún tipo de cáncer.

Como dijimos, en todo el parque Sarmiento, ya se están realizando deportes. Por lo que la propuesta contempla una tipología de dispositivo que colabore para realizar en forma más completa y confortable el ejercicio físico. El proyecto consiste en un volumen que muta, que se despliega para dar respuesta a muchas funciones requeridas.

Siempre se encuentran en parques de distintas ciudades, en situaciones domésticas, kioskos y pabellones transitorios. La microarquitectura aquí propuesta se complementa con dos elementos: una estructura cilíndrica y unos gajos, que son parte de ese cilindro que se despliegan para dar lugar a las funciones.

Todo el conocimiento acumulado sobre estas relaciones triangulares, del edificio al individuo, del individuo a la naturaleza y de la naturalezas al edificio, vendrían a formar el core del conocimiento estructurado sobre lo que ampliamente llamamos arquitectura, (Aurelli, 2012)

La piel metálica que envuelve la arquitectura y le permite personalizarlo. Los elementos que acompañan a la microarquitectura y que combinados entre ellos definen diferentes configuraciones son:

- Bancos de descanso y barras de elongación.

- Área de hidratación, con filtros de agua.

- Área de reposición de energías con semillas y frutas.

- Soporte para bicicletas.

- Espacio verde/ regado/ techo verde.

Debemos respetar las características que ya definimos para micro-arquitectura urbana son:

- Estética.

- Seguridad, se tiene que poder cerrar, antivandalismo.

- Solidez y duración.

- Funcionalidad.
- Idoneidad de instalación.

- Facilidad de reparación.

- Facilidad de mantenimiento.

- Acordar con la ordenanza municipal.

\section{Características intrínsecas del dispositivo:}

Tenemos como objetivo trabajar para la ciudad ofreciendo al usuario ciudadano:
a. Protección y estar confortable.
b. Información y comunicación.
c. Reposo e higiene.
d. Interacción del usuario con los objetos y el espacio.

Si continuamos con las características de esta tipología, decimos que no es temporario, podría ser repetible, para distintas áreas.

Mantenemos el objetivo de que toda construcción debe ser sustentable e inclusiva, respetando las necesidades del adulto mayor y del niño.

Trabajar no sólo para satisfacer necesidades primarias, sino para desarrollar espacios confortables.

Córdoba tiene muchas jornadas de más de 35 grados, para la propuesta d hidratación, podríamos entregar agua filtrada y fresca?

Consideramos el clima, temperaturas extremas de verano e invierno, lluvia, etc. para el diseño, que tendrá techos verdes, desagües de lluvia del dispositivo y del área, y la posibilidad de recolección de la misma para mantener el área verde.

Se estudiará en una segunda etapa las preexistencias vientos y ruido, diseñando protectores de vientos, orientación del parador, etc.

Se propone una combinación de usos: parador de bicicleta, residuos, contenedor de verde, (cazuela de árbol, maceteros, espacio verde), bebedero, semillas, frutas, papelero, cargador de celular, reciclado de botellas platicas, etc.

Sistemas, de seguridad con cerramiento total y cámaras, de señalética, luminosa, de señalética urbana turística, nube, de señalética comercial, publicidad. Se incluirá el diseño de la Señalética integral, la del sistema urbano, la turística y la comercial; publicidad, tipo, sistemas posibles, etc. 
Contemplaremos sistemas generadores de energía, para iluminación y para el cargador de refrigeración de frutas.
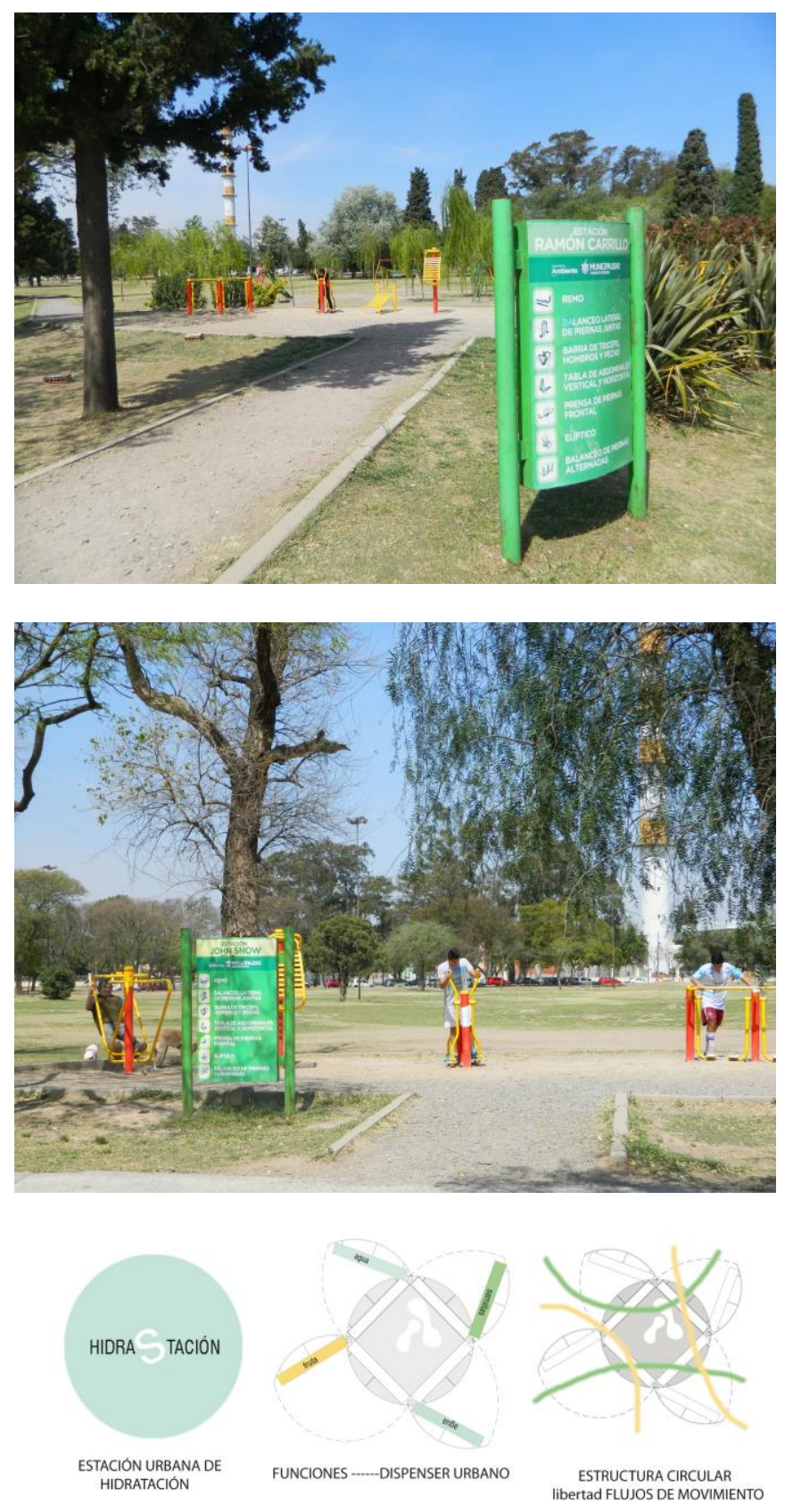

\section{Conclusión y Discusión}

Trabajamos sobre ciertos mitos que estamos convencidos podemos desterrarlos, basadas en investigaciones anteriores.

Es posible trabajar con tipologías para el municipio, de servicio, que sean inclusivas, sustentables y accesibles económicamente con tecnologías domóticas?
Hasta qué punto el usuario prefiere interactuar con el espacio y los equipos? Cuál es el grado o funciones en que prefiere decidir por sí mismo o elige que los sistemas se lo resuelvan.

Es un desafío para este grupo trabajar desde lo micro, equipamientos, a escala mayores y complejas como son los espacios de la ciudad. Se plantea con esta investigación el desafío de regenerar sectores, de considerar actividades y proyectar para ellas con tecnologías de vanguardia, en una sinergia sostenible e inclusiva, respetando las preexistencias espaciales.
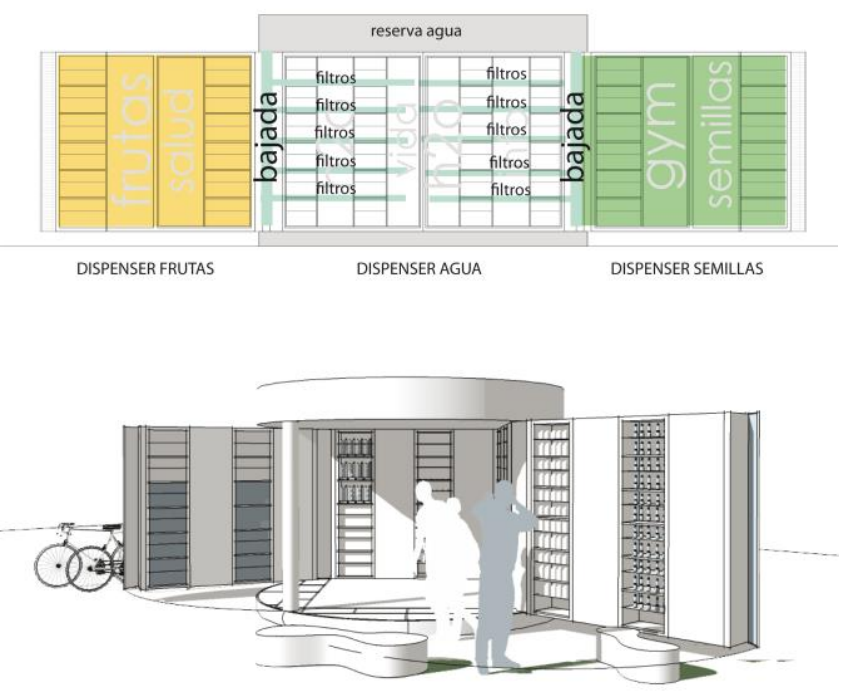

HIDROSTACIÓN --.--MICROARQUITECTURA URBANA

Imagen 7 y 8 , imágenes propias.

\section{Agradecimientos}

Agradecemos a los asesores Ingenieros, Lucio Fernando Madussi, (lucholemm@gmail.com); Hugo Dallegre(hdallegre@gmail.com); y al ing Marcos Blasco,(marcos.ielect@gmail.com) de la facultad de CEFyN UNC .

\section{Bibliografía}

Ábalos, I. y Herrreros, J..(1997.) Areas de Impunidad. Editorial Actar, Barcelona.

Augé, M.1993), Los no lugares, Espacios de anonimato. Una antropología de la sobremodernidad. Editorial Gedisa. Barcelona.

AURELI, Pier Vittorio (2012), The Common and the Production of Architecture, Early Hypotheses, en Common Ground, A Critical 
SIGraDi 2016, XX Congreso de la Sociedad Ibero-americana de Gráfica Digital

9-11, November, 2016 - Buenos Aires, Argentina

Reader, Ed. David Chiperfield, Kieran Long and Shumi Bose, Marsilio Editori, Venezia,

BOHIGAS, Oriol. Reconstrucció de Barcelona. Barcelona: Edicions 62, 1985. 302 p. Trad. al cast. Reconstrucción de Barcelona. Madrid: MOPU, 1986.

Batty, M. (2005). Cities and complexity: Understanding cities through cellular automata, agent-based models, and fractals. Cambridge, MA: The MIT Press

CEDOM (2007) Asociación Española de Domótica. Cuaderno de divulgación Domótica. $2{ }^{a}$ ed. España, Barcelona: Aenor

Cabezas, C. (2014) "Claves Para Proyectar Espacios Públicos Confortables. Indicador del confort en el espacio público" 05 Jun 2014. ArchDaily. Recuperado 15 Sep 2014. http://www.plataformaarquitectura.cl/cl/02-285882/claves-paraproyectar-espacios-publicos-confortables-indicador-del-confort-enel-espacio-publico.

Diccionario Espasa Calpe (1945), Espasa Calpe Editora ,2da Edición Argentina, Buenos Aires, México -

Deleuze, G.y Guattari, F.. Mil mesetas. Capitalismo y Esquizofrenia. Ed. De Minuit. Paris, 1980.

E2 groupe. E2 - Exploring the Urban condition. Editorial ACTAR. Paris 2003.

Fariña, F.S. (2006/2008) Predios Urbanos Intersticiales (PUInt). Validación de Herramienta de Análisis Diagnóstico Antecedente1 y
Generación de Hipótesis Teóricas Fundantes de Procesos Proyectuales IDEHAB - Facultad de Arquitectura y Urbanismo SeCyT - Universidad Nacional de La Plata. Director: Arq. V. Schaposnik.

Gonzalo G. E., et al. (2007). Diseño Bioclimático de Oficinas. Pautas para San Miguel de Tucumán. Centro de Estudios Energía y Medio Ambiente, Instituto de Acondicionamiento Ambiental. Tucumán: el autor.

Hernández S. P. et al (2008) microarquitectura Equipo domotizado para auto-consulta en librería http://cumincades.scix.net/data/works/att/sigradi2008_043.content. pdf

Koolhass, R - (2000) El espacio basura, en ARQUITECTURA VIVA № 74 . Madrid.

Monroy, Manuel Martin. Manual de Diseño ICARO de Calidad Ambiental en la Edificación. Departamento de Construcción Arquitectónica de la Universidad de Las Palmas de la Gran Canaria (2006).

Recuero, A. (1999) Informes de la Construcción, Vol. 50 n 459.

Schumacher, P. (2008). Digital Cities. AD Architectural Design. Parametricism. A New Global Style for Architecture and Urban Design. 79 (4) 14-23.

STOPPANI, Teresa(2012) , A Conversation of Many, en Common Ground, A Critical Reader, Ed. David Chiperfield, Kieran Long and Shumi Bose, Marsilio Editori, Venezia, 